\title{
CD26/dipeptidyl peptidase IV enhances expression of topoisomerase II alpha and sensitivity to apoptosis induced by topoisomerase II inhibitors
}

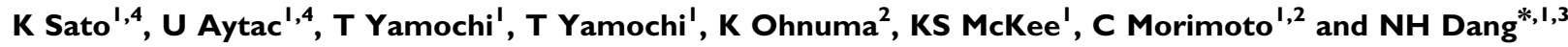 \\ 'Department of Lymphoma/Myeloma, MD Anderson Cancer Center, 1515 Holcombe Boulevard, Houston, TX 77030, USA; '2Department of Clinical \\ Immunology and AIDS Research Center, Institute of Medical Science, University of Tokyo, 4-6-I, Shirokanedai, Minato-ku, Tokyo I08-8639, Japan; \\ ${ }^{3}$ Department of Molecular Therapeutics, MD Anderson Cancer Center, 1515 Holcombe Boulevard, Houston, TX 77030, USA
}

\begin{abstract}
CD26/dipeptidyl peptidase IV (DPPIV) is a cell surface-bound ectopeptidase with important roles in T-cell activation and tumour biology. We now report that CD26/DPPIV enhances sensitivity to apoptosis induced by the antineoplastic agents doxorubicin and etoposide. In particular, CD26/DPPIV presence is associated with increased susceptibility to the mitochondrial pathway of apoptosis, documented by enhanced cleavage of poly (ADP ribose) polymerase (PARP), caspase-3 and caspase-9, Bcl-xl, and Apaf-I, as well as increased expression of death receptor 5 (DR5). We also show that the caspase-9-specific inhibitor z-LEHD-fmk inhibits drugmediated apoptosis, leading to decreased PARP and caspase-3 cleavage, and reduced DR5 expression. Importantly, through detailed studies that demonstrate the association between topoisomerase II alpha expression and DPPIV activity, our data provide further evidence of the key role played by CD26 in biological processes.

British Journal of Cancer (2003) 89, 1366 - 1374. doi:I0.1038/sj.bjc.660 I 253 www.bjcancer.com
\end{abstract}

(c) 2003 Cancer Research UK

Keywords: CD26/DPPIV; apoptosis; topoisomerase II; caspase-9; DNA damage

CD26 is a $110-\mathrm{kDa}$ type II cell surface glycoprotein with diverse functional properties that is widely expressed on various tissues (Dang and Morimoto, 2002; Sato and Dang, 2003). Its extracellular domain encodes a membrane-associated dipeptidyl peptidase IV (DPPIV) activity capable of processing amino-terminal dipeptides from polypeptides with either L-proline or L-alanine in the penultimate position (Oravecz et al, 1997). In addition, CD26 acts as an alternative pathway of T-cell activation through its physical and functional association with molecules involved in T-cell signal transduction processes, including CD45, mannose 6-phosphate/ insulin-like growth factor II receptor and adenosine deaminase (ADA) (Morimoto et al, 1989; Dang et al, 1990a-d, 1991; Ishii et al, 2001; Dang and Morimoto, 2002; Sato and Dang, 2003). With its deficiency being a cause of severe combined immune deficiency, ADA plays a key role in the development and function of lymphoid tissues and by regulating surface expression of ADA, CD26 potentially has an important role in adenosine metabolism and immune regulation (Sato and Dang, 2003). Meanwhile, recent findings suggest that CD26 has a role in the development of certain types of neoplasms (Morrison et al, 1993; Sedo and Revoltella, 1995; Tanaka et al, 1995; Stecca et al, 1997). B-chronic lymphocytic leukaemia cells have high levels of CD26 protein expression and mRNA transcripts (Bauvois et al, 1999); whereas the more

*Correspondence: Dr NH Dang, MD Anderson Cancer Center, BOX 429, 1515 Holcombe Boulevard, Houston, TX 77030, USA;

E-mail: nhdang@mail.mdanderson.org

${ }^{4}$ Contributed equally to this work.

Received 28 March 2003; revised I5 July 2003; accepted I5 July 2003 aggressive T-cell malignancies, such as T-cell acute lymphoblastic leukaemia or $\mathrm{T}$-cell $\mathrm{CD} 30+$ anaplastic large-cell lymphoma, express higher CD26 level as compared to the more indolent $\mathrm{T}$ cell diseases like mycosis fungoides (Carbone et al, 1995; Jones et al, 2001).

Topoisomerase II inhibitors are widely used agents in cancer treatment (Froelich-Ammon and Osheroff, 1995), exploiting the catalytic activity of topoisomerase II alpha by increasing permanent DNA damage (Beck et al, 1999). Previous findings have shown that DNA damage mediated by topoisomerase II inhibitors induces apoptosis (Beck et al, 1999; Mow et al, 2001), particularly through cytochrome $c$ release (Liu et al, 1996; Kluck et al, 1997), Apaf-1 involvement (Perkins et al, 2000; Lauber et al, 2001), and subsequent caspase-9 activation (Mow et al, 2001). We have previously shown that surface expression of CD26/DPPIV enhances sensitivity of CD26 Jurkat T-cell transfectants to G2/M arrest mediated by the topoisomerase II inhibitors doxorubicin and etoposide (Aytac et al, 2001, 2003). Expanding on these previous findings, we now examine the effect of CD26/DPPIV expression on apoptosis induced by doxorubicin and etoposide. In this paper, we demonstrate that CD26/DPPIV presence enhances sensitivity to apoptosis induced by topoisomerase II inhibitors, associated with increased cleavage of Bcl-xl, Apaf-1, procaspase-9, procaspase-3, and PARP, as well as increased expression of DR5. Meanwhile, pretreatment with the caspase-9-specific inhibitor z-LEHD-fmk significantly reduces etoposide-mediated apoptotic events. Importantly, we present detailed data from different experimental approaches demonstrating the association between DPPIV enzyme activity and topoisomerase II alpha expression. Our findings thus emphasise the increasingly important role of the multifaceted 
CD26/DPPIV molecule in biological processes, while the functional association between CD26/DPPIV and topoisomerase II alpha may be exploited for future treatments of selected cancers.

\section{MATERIALS AND METHODS}

\section{Cells and reagents}

Human CD26 Jurkat T-cell leukaemia stable transfectants have been described and characterised previously regarding CD26 surface expression and associated DPPIV enzyme activity (Aytac et al, 2001, 2003). The Jurkat cell lines include: (a) wild-type CD26transfected Jurkat cell lines (wtCD26); (b) Jurkat cell lines transfected with mutant CD26 containing an alanine at the putative catalytic serine residue at position 630 , resulting in a mutant CD26positive/DPPIV-negative Jurkat transfectant (S630A); (c) Jurkat cell lines transfected with mutant CD26 containing point mutations at ADA-binding site residues 340-343, with amino acid L340, V341, A342, and R343 being replaced by amino acids P340, S341, E342, and Q343, resulting in a mutant CD26-positive/DPPIV-positive Jurkat transfectant incapable of binding ADA (340-344); and (d) nontransfected control Jurkat cells (parental). Jurkat transfectants were maintained in culture media, which consisted of RPMI 1640 supplemented with $10 \% \mathrm{FCS}$, penicillin $\left(100 \mathrm{U} \mathrm{ml}^{-1}\right)$, streptomycin $\left(100 \mu \mathrm{g} \mathrm{ml}^{-1}\right)$, and G418 $\left(0.25 \mathrm{mg} \mathrm{ml}^{-1}\right.$; Life Technologies Inc., NY, USA) Nontransfectant control Jurkat cells were maintained in the same culture media without G418. Annexin V- fluorescein isothiocyanate (FITC) was from BD PharMingen, CA, USA. Anti-PARP, cytochrome $c$, and caspase- 3 Abs were from BD PharMingen; anti-actin was from Sigma Chemical Co, MO, USA; anti-caspase- 9 was from Cayman, MI, USA. Anti-DR5 Abs were from Cayman. Anti-Bcl-xl and Apaf-1 Abs were from BD Transduction Laboratories, CA, USA. Anti-topoisomerase II alpha was from Roche, IN, USA. Caspase-9 inhibitor (z-LEHD-fmk) was from BD PharMingen. Diisopropyl fluorophosphate (DFP) was obtained from SIGMA, MO, USA. Substrate for DPPIV, Gly-Pro- $p$-nitroanilide-tosylate (GPNT), was purchased from WAKO, Japan. Etoposide was purchased from SIGMA and was dissolved in sterile DMSO. Doxorubicin was purchased from Calbiochem, CA, USA and was dissolved in sterile PBS. Soluble CD26 molecules were produced by Chinese hamster ovary cells and purified as described previously (Tanaka et al, 1994).

\section{Annexin/propidium iodide (PI) assays}

Exposure of phosphatidylserine residues was quantified by surface Annexin V staining as previously described (Raynal and Pollard, 1994). Briefly, cells were washed in binding buffer (10 mM HEPES, $\mathrm{pH}$ 7.4, $2.5 \mathrm{~mm} \mathrm{CaCl}, 140 \mathrm{~mm} \mathrm{NaCl}$ ), resuspended in $100 \mu \mathrm{l}$ and incubated with $0.5 \mu \mathrm{lml}^{-1}$ annexin V-FITC and $2.5 \mu \mathrm{g} \mathrm{ml}^{-1}$ PI for $15 \mathrm{~min}$ in the dark. Cells were then washed again and resuspended in $400 \mu \mathrm{l}$ of binding buffer, then flow cytometric analysis (FACScan; Becton Dickinson, CA, USA) was performed. A total of 10000 cells were acquired per sample and data were analysed using Cellquest software (Becton Dickinson).

\section{SDS-PAGE and immunoblotting}

After incubation at $37^{\circ} \mathrm{C}$ in culture media and etoposide or doxorubicin at the concentrations and duration indicated, cells were harvested from wells, washed with PBS, and lysed in lysis buffer consisting of $1 \% \mathrm{NP}-40,0.5 \%$, deoxycolate, $0.1 \%$ SDS, $1 \mathrm{~mm}$ phenylmethylsulphonyl fluoride, $1 \mathrm{~mm}$ benzamidine, $10 \mu \mathrm{g} \mathrm{ml}^{-1}$ aprotinin, $50 \mu \mathrm{g} \mathrm{ml}^{-1}$ leupeptin, $10 \mu \mathrm{g} \mathrm{ml}^{-1}$ soybean trypsin inhibitor and $1 \mu \mathrm{g} \mathrm{ml}^{-1}$ pepstatin. After incubating on ice for $5 \mathrm{~min}$, nuclei were removed by centrifugation and supernatants were collected as whole-cell lysates. Sample buffer $(4 \times)$ consisting of $20 \%$ glycerol, $4.6 \%$ SDS, $0.5 \mathrm{M}$ Tris ( $\mathrm{pH}$ 6.8), $4 \% \quad \beta$ - mercaptoethanol, and $0.2 \%$ bromophenol blue was added to the appropriate aliquots of supernatants. After boiling, protein samples were submitted to SDS-PAGE analysis on an $8 \%$ gel under standard conditions using a mini-Protean II system (BioRad, CA, USA). For immunoblotting, the proteins were transferred onto nitrocellulose (Immobilon-P; Millipore, MA, USA). After overnight blocking at $4{ }^{\circ} \mathrm{C}$ in blocking solution consisting of $0.1 \%$ Tween 20 and 5\% BSA in Tris-buffered saline, membranes were blotted with the appropriate primary antibodies diluted in blocking solution for $1 \mathrm{~h}$ at room temperature. Membranes were then washed with blocking solution, and appropriate secondary antibodies diluted in blocking solution were then applied for $1 \mathrm{~h}$ at room temperature. Secondary antibodies were goat anti-mouse or goat anti-rabbit horseradish peroxidase conjugates (Dako). Membranes were then washed with blocking solution, and proteins were subsequently detected by chemiluminescence (Amersham Pharmacia Biotech, NJ, USA).

\section{Dipeptidyl Peptidase IV enzyme activity assays}

As previously described (Kajiyama et al, 2002), DPPIV enzyme activity was measured spectrophotometrically using GPNT, a substrate for DPPIV. A $1 \times$ PBS-washed whole-cell suspension was prepared and $5 \times 10^{5}$ cells were resuspended in $200 \mu$ l of PBS into 96-well plate, then GPNT was added at a final concentration of $0.24 \mathrm{~mm}$. The absorption was measured at $405 \mathrm{~nm}$ using microplate spectrophotometer (BIO-TEK Instruments, inc., VE, USA) twice, just before the addition of the substrate and after $60 \mathrm{~min}$ incubation at $37^{\circ} \mathrm{C}$. Dipeplidyl peptidase enzyme activity was calculated from the increase of absorption between 0 and $60 \mathrm{~min}$.

\section{Inhibition of DPPIV enzyme activity}

As described previously (Koreeda et al, 2001; Kajiyama et al, 2002), DFP was used as the DPPIV chemical inhibitor for inhibition assays. To evaluate effect of continuous exposure to DFP, wtCD26 transfectants or parental Jurkat cells were incubated in culture media alone (DFP-), culture media containing $100 \mu \mathrm{M}$ DFP for $2 \mathrm{~h}$ or for $6 \mathrm{~h}(\mathrm{DFP}+)$. A representative sample of cells reflecting each treatment condition was obtained for DPPIV enzyme activity assays or to examine topoisomerase II alpha expression. Alternatively, wtCD26 Jurkat transfectants were incubated in culture media; or in culture media with $100 \mu \mathrm{M}$ DFP for $4 \mathrm{~h}$; or they were incubated in culture media with $100 \mu \mathrm{M}$ DFP for $4 \mathrm{~h}$, then washed twice in PBS to ensure removal of DFP followed by incubation in culture media for 2 or $8 \mathrm{~h}$. A representative sample of cells reflecting each treatment condition was obtained for DPPIV enzyme activity assays or to examine topoisomerase II alpha expression. For all treatment conditions, trypan blue uptake assays consistently showed $>90 \%$ cell viability (data not shown).

\section{Preparation of cytosol fractions}

As previously described (Haridas et al, 2001; Nishimura et al, 2001), Jurkat cells $\left(4.0 \times 10^{7}\right)$ were suspended in $1 \mathrm{ml}$ sucrose buffer (250 mm sucrose in $30 \mathrm{~mm}$ Tris $\mathrm{HCl}, \mathrm{pH} 7.4)$ and transferred into an $\mathrm{N}_{2}$ cavitation chamber (PARR Instruments, Moline, IL, USA). The cells were subjected to $\mathrm{N}_{2}$ cavitation ( 250 psi for $5 \mathrm{~min}$ ) according to the manufacturer's instructions. Under these conditions, most of the cell membrane was disrupted with no change in the mitochondrial respiratory activity. Next, DNA and the nuclear fraction were removed by centrifugation $(1500 \mathrm{~g}$ for $2 \mathrm{~min})$. The supernatant was further centrifuged $(16000 \mathrm{~g}$ for $10 \mathrm{~min})$, and the supernatant was used as the cytosol fraction.

\section{Preparation of nuclear extracts}

Cells $\left(10 \times 10^{6}\right)$ were harvested and allowed to swell for $15 \mathrm{~min}$ on ice in cytoplasmic extraction buffer (10 mM HEPES, $10 \mathrm{~mm} \mathrm{KCl}$, 
$0.1 \mathrm{~mm}$ EDTA, 0.1 mm EGTA, $1 \mathrm{~mm}$ DTT, $1 \mathrm{~mm}$ PMSF, $2 \mu \mathrm{g} \mathrm{ml}^{-1}$ leupeptin, $2 \mu \mathrm{g} \mathrm{ml}^{-1}$ aprotinin, and $0.5 \mathrm{mg} \mathrm{ml}^{-1}$ benzamidine). Then NP-40 (final concentration 0.3\%) was added into that cell suspension and vortexed for $10 \mathrm{~s}$. After 2 min-centrifugation at $16000 \mathrm{~g}$, the supernatant was discarded. The pellet was then incubated with nuclear extraction buffer $(20 \mathrm{~mm}$ HEPES, $400 \mathrm{~mm}$ $\mathrm{KCl}, 1 \mathrm{~mm}$ EDTA, $1 \mathrm{~mm}$ EGTA, $1 \mathrm{~mm}$ DTT, $0.5 \mathrm{~mm}$ PMSF, $2 \mu \mathrm{g} \mathrm{ml}^{-1}$ leupeptin, $2 \mu \mathrm{g} \mathrm{ml}^{-1}$ aprotinin, and $0.5 \mathrm{mg} \mathrm{ml}^{-1}$ benzamidine) for $30 \mathrm{~min}$ on ice with intermittent vortexing. The suspension was centrifuged at $16000 \mathrm{~g}$ for $6 \mathrm{~min}$, and the supernatant was saved as the nuclear extract.

\section{RESULTS}

\section{Effect of CD26/DPPIV expression on apoptosis of Jurkat cells mediated by topoisomerase II inhibitors}

Annexin V/PI assays show that wtCD26 Jurkat transfectants are more sensitive to the apoptotic effect of etoposide than S630A or parental control Jurkat cells. Meanwhile, 340-4 transfectants $(340-4)$ exhibit higher level of drug-induced apoptosis, similar to that of wtCD26 transfectants (Figure 1A). Furthermore, wtCD26 and 340-4 cells display greater apoptosis when treated with doxorubicin as compared with parental or S630A Jurkat cells (Figure 1B). Time course studies to evaluate the effect of doxorubicin on apoptosis in these CD26 Jurkat transfectants are also performed. Our data show that wtCD26 Jurkat transfectants consistently exhibit greater drug-induced apoptosis over the time intervals and drug concentrations tested than parental Jurkat (Figure 1C), strongly suggesting that the presence of CD26 directly enhances cellular sensitivity to topoisomerase II inhibitor-induced apoptosis. Similar results are obtained with etoposide treatment (data not shown). Similarly, both the wtCD26 and 340-4 Jurkat transfectants are more sensitive to etoposide-mediated PARP cleavage (Figure 2), as compared with parental cells and S630A transfectants. Similar results are seen when cells are treated with doxorubicin. These data hence suggest that the presence of CD26, especially its associated DPPIV enzymatic activity, enhances apoptosis mediated by topoisomerase II inhibitors.

\section{Effect of CD26/DPPIV surface expression on the mitochondrial pathway of apoptosis induced by etoposide}

Previous work demonstrated that DNA damage mediated by topoisomerase II inhibitors induces apoptosis through the mitochondrial pathway. Our time course analyses (Figure 3 ) show that etoposide treatment results in enhanced cleavage of PARP and procaspase-3, leading to increased levels of the cleaved $17 \mathrm{kDa}$ caspase-3 bands in wtCD26 transfectants, as compared with S630A

Figure I Enhancing effect of CD26/DPPIV surface expression on apoptosis induced by topoisomerase II inhibitors. CD26 Jurkat transfectants were incubated at $37^{\circ} \mathrm{C}$ in culture media alone or culture media containing etoposide (A) for $14 \mathrm{~h}$ or doxorubicin (B) for $16 \mathrm{~h}$ at the concentrations indicated. Cells were then harvested and Annexin V/PI assays were performed as described in Materials and Methods. wtCD26: wild-type CD26 Jurkat transfectant; S630A: Jurkat cells transfected with mutant CD26 containing an alanine at the putative catalytic serine residue at position 630 , resulting in a mutant CD26-positive/DPPIV-negative Jurkat transfectant; control: nontransfected parental Jurkat; 340-4: Jurkat cells transfected with mutant CD26 containing point mutations at the ADA-binding site residues 340 - 343 , with amino acids $L_{340}, V_{341}, A_{342}$, and $R_{343}$ being replaced by amino acids $P_{340}, S_{34}, E_{342}$, and $Q_{343}$, resulting in a mutant CD26-positive/ DPPIV-positive mutant CD26 Jurkat transfectant incapable of binding ADA. Data are representative of three separate experiments. $(\mathbf{C})$ wtCD26 Jurkat transfectants and parental cells were treated with doxorubicin over the indicated time intervals and drug concentrations. a: $12 \mathrm{~h}, \mathrm{~b}: 24 \mathrm{~h}, \mathrm{c}: 36 \mathrm{~h}$ Data are representative of three separate experiments. and parental Jurkat cells. Moreover, our work shows that etoposide treatment leads to significantly greater cleavage of procaspase- 9 in wtCD26 cells compared to S630A and parental control Jurkat. We also demonstrate greater cleavage of the $130 \mathrm{kDa}$ proform of Apaf1 in etoposide-treated wtCD26 Jurkat transfectants as compared with parental control or S630A Jurkat. Furthermore, the increase in sensitivity to etoposide-induced apoptosis in wtCD26 transfectants is accompanied by greater cleavage of the full-length antiapoptotic

\section{A}
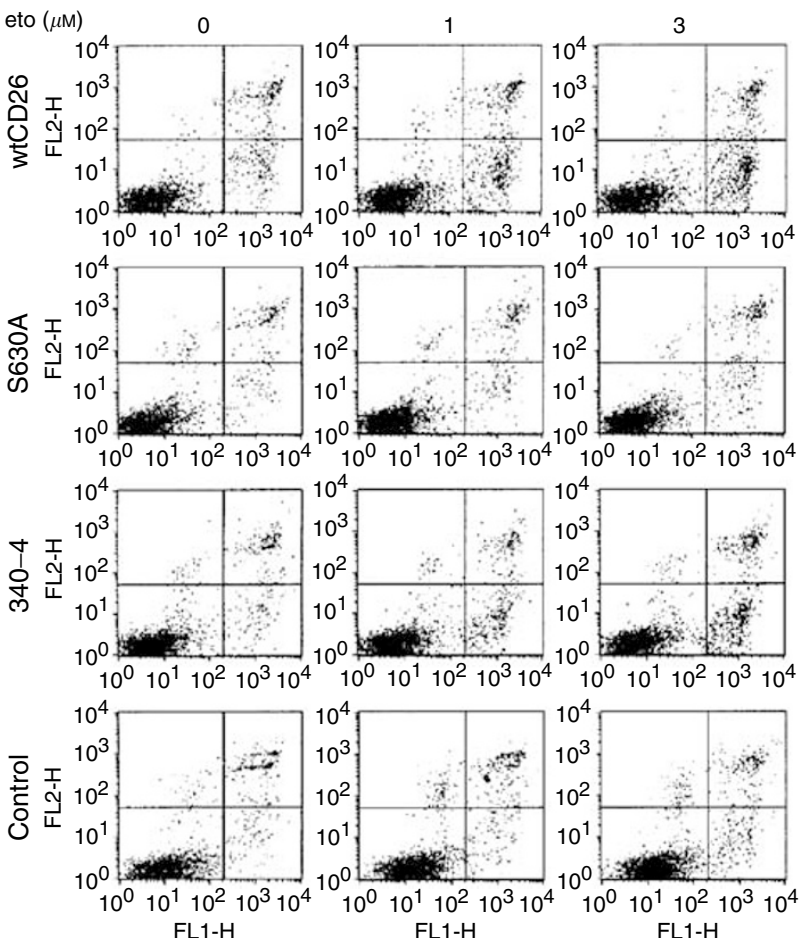

B
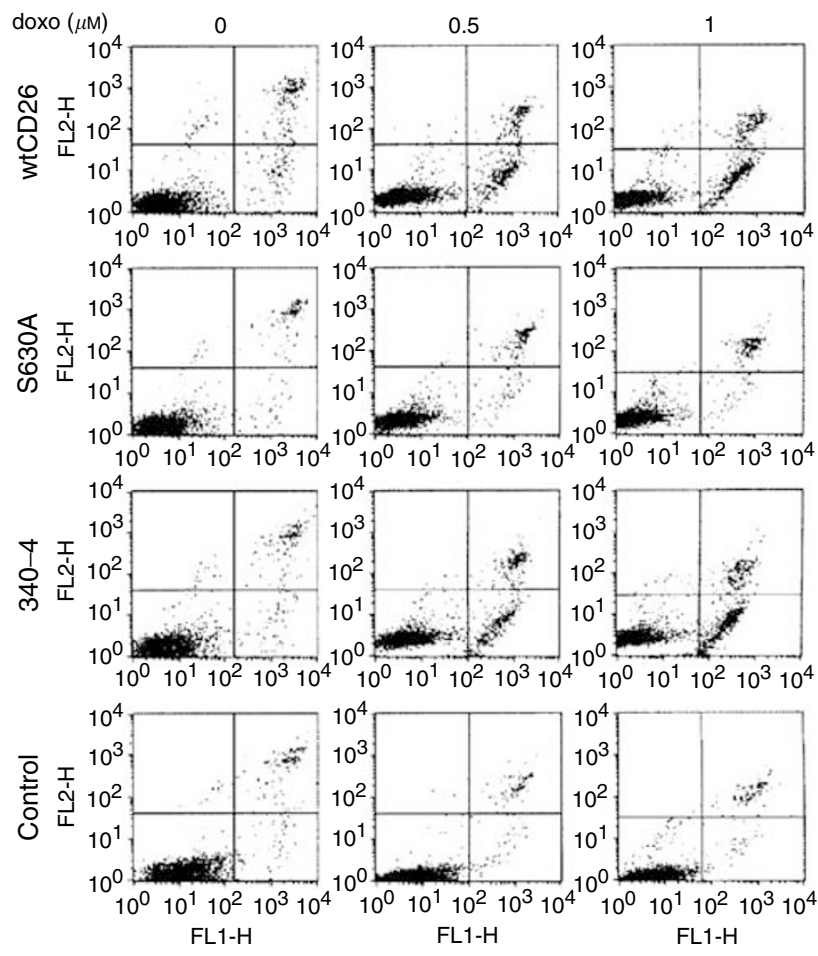
C
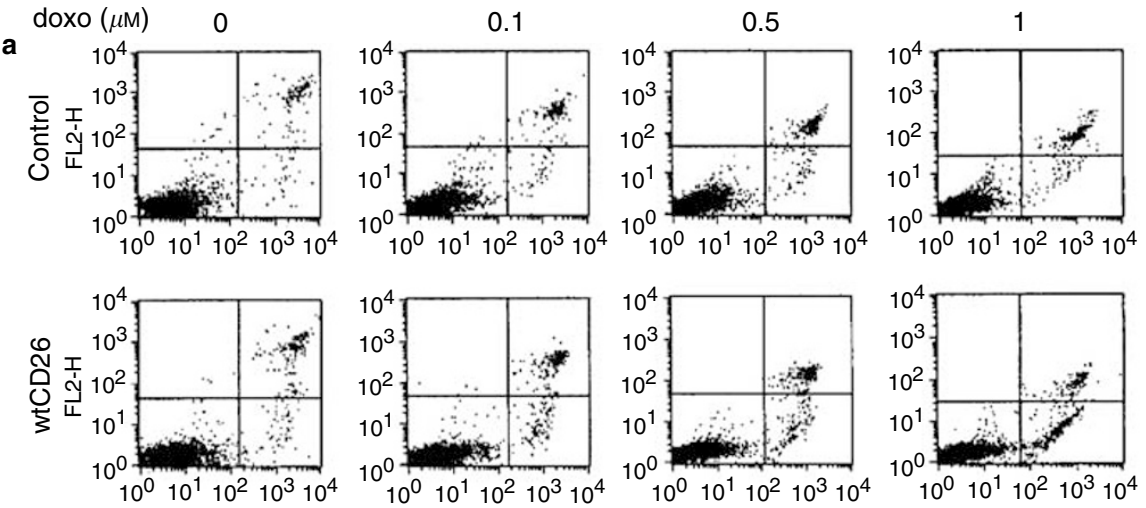

b
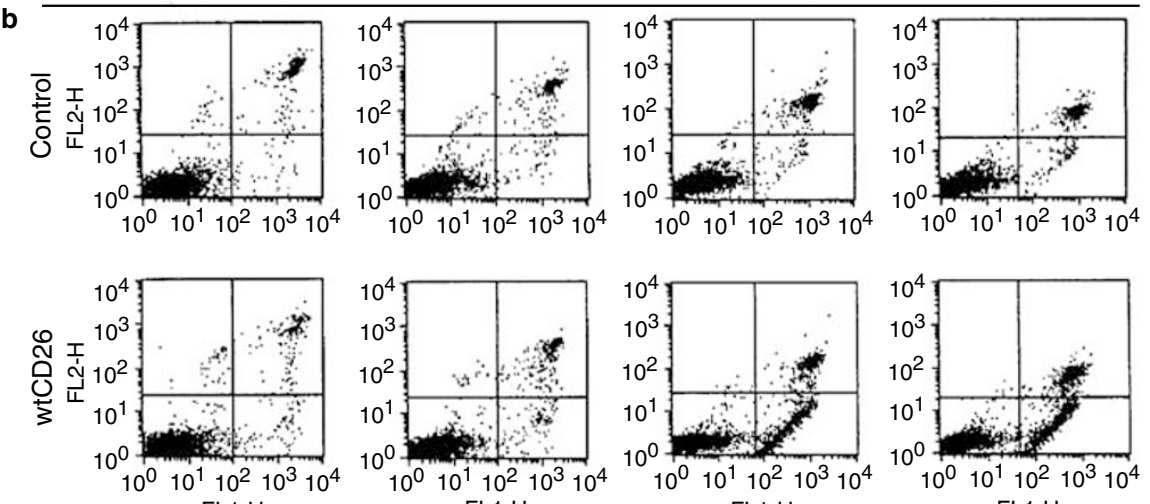

C
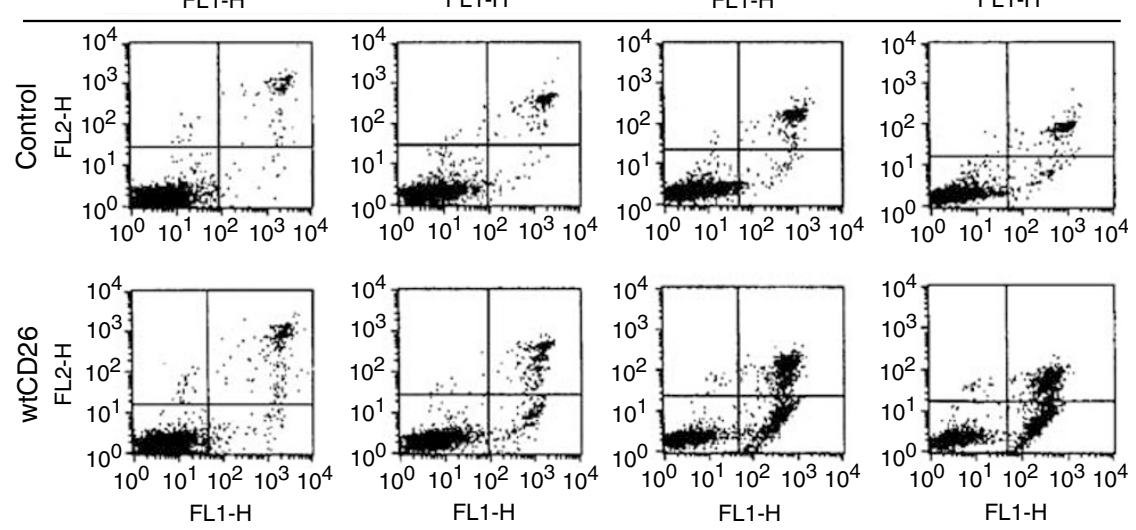

Figure I Continued.

molecule Bcl-xl (Fujita et al, 1998) and a resultant rise in the $18 \mathrm{kDa}$ cleaved band. Taken together, our results indicate that CD26/DPPIV enhances etoposide-mediated apoptosis of Jurkat cells by affecting cellular processes known to be involved in drugmediated apoptosis, including those involving caspase- 9 processing and the mitochondrial pathway, as well as processing of bcl-2related molecules. Similarly, time course analyses demonstrate that wtCD26 Jurkat transfectants exhibit greater apoptosis through caspase- 9 processing when treated with doxorubicin, as demonstrated by greater cleavage of PARP and procaspase- 9 .

Effect of the caspase-9 inhibitor z-LEHD-fmk on etoposideinduced apoptosis in CD26 Jurkat transfectants

To further confirm our findings that CD26 affects etoposideinduced apoptosis through caspase-9-related events, we evaluated the effect of the caspase- 9 inhibitor z-LEHD-fmk on this process. Western blot analyses show that pretreatment with z-LEHD-fmk significantly abrogates the effect of etoposide on wtCD26 Jurkat transfectants. As shown in Figure 4, etoposide-mediated cleavage of procaspase- 9 is inhibited by z-LEHD-fmk in a dose-dependent manner. Furthermore, cleavage of procaspase- 3 and PARP, events downstream of caspase- 9 processing, is significantly reduced following pretreatment with the caspase- 9 inhibitor. Our data therefore indicate CD26 augments etoposide-induced apoptosis in CD26 Jurkat transfectants through caspase-9-related events. Similarly, pretreatment with z-LEHD-fmk significantly decreases the effect of doxorubicin on wtCD26 Jurkat transfectants, as measured by cleavage of PARP and procaspase-9.

\section{Effect of the DPPIV enzyme inhibitor DFP on topoisomerase II alpha expression}

We previously showed that topoisomerase II alpha expression and catalytic activity are higher in wtCD26 Jurkat transfectant than S630A or parental cells (Aytac et al, 2003) (Figure 5A). To further 


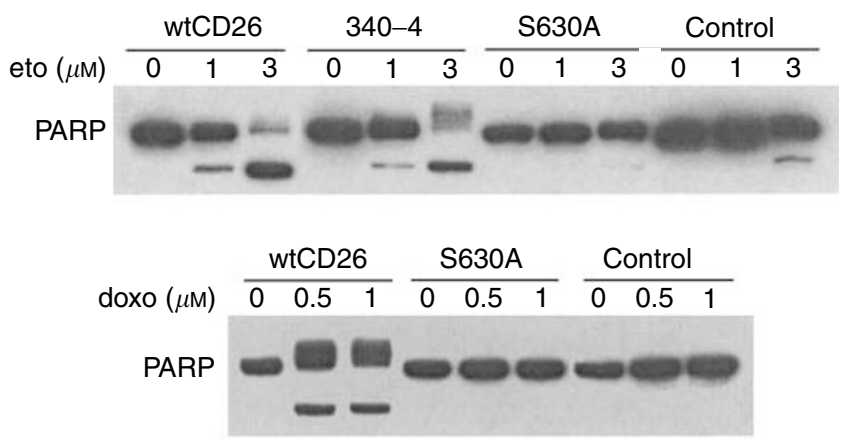

Figure 2 CD26/DPPIV-associated enhancement in PARP cleavage induced by topoisomerase II inhibitors. CD26 Jurkat transfectants were incubated at $37^{\circ} \mathrm{C}$ with media containing etoposide for $16 \mathrm{~h}$ or doxorubicin for $18 \mathrm{~h}$ at the indicated doses. Cells were then harvested, and whole-cell lysates were obtained. Following SDS-PAGE of lysates, immunoblotting studies for PARP and $\beta$-actin were performed as described in Materials and Methods. The cleaved product of PARP was detected at $\sim 85 \mathrm{kDa}$. Each lane was loaded with $30 \mu \mathrm{g}$ of protein

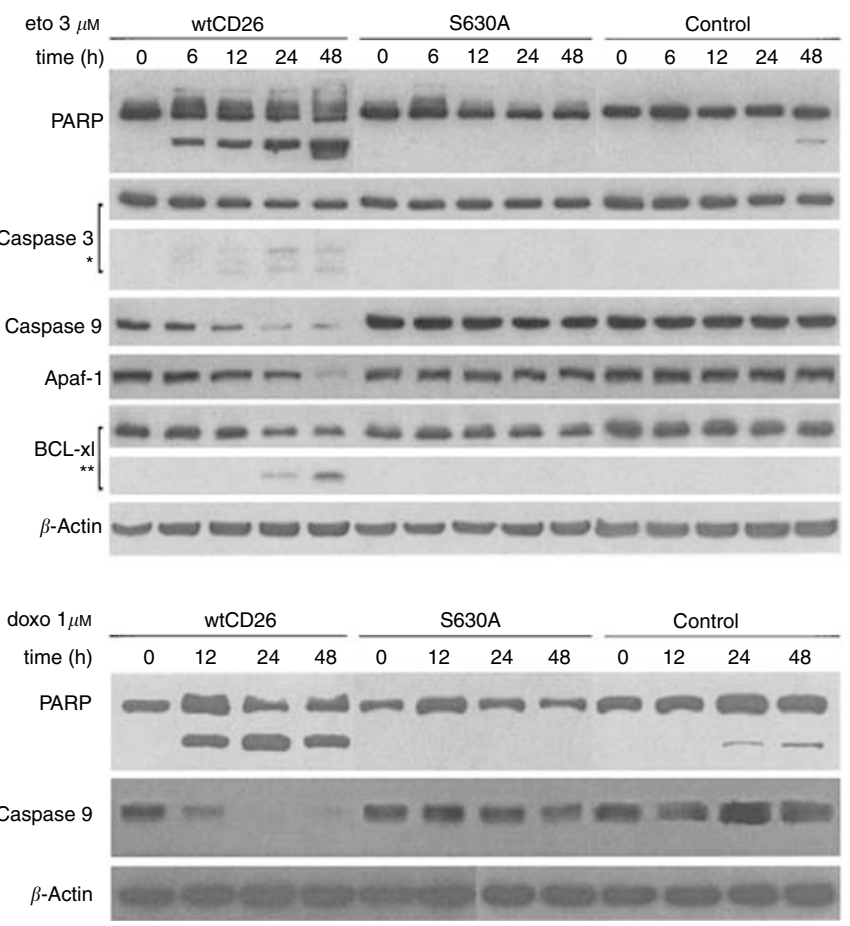

Figure 3 Time course study of the effect of CD26/DPPIV surface expression on etoposide-induced apoptosis. Jurkat cells were incubated at $37^{\circ} \mathrm{C}$ with media containing $3 \mu \mathrm{M}$ etoposide or I $\mu \mathrm{M}$ doxorubicin for the indicated time periods at the indicated doses. Cells were then harvested, and cytosol fractions were obtained as described in Materials and Methods. Following SDS-PAGE of lysates, immunoblotting studies with specific antibodies for PARP, caspase-9, caspase-3, Apaf-I, Bcl-xl, and $\beta$-actin were performed as described in Materials and methods (*): caspase-3 cleaved products; (**): Bcl-xl cleaved products. Each lane was loaded with $30 \mu \mathrm{g}$ of protein.

evaluate in detail the effect of DPPIV activity on topoisomerase II alpha expression, we examined the effect of the DPPIV chemical inhibitor DFP (Koreeda et al, 2001; Kajiyama et al, 2002) on topoisomerase II alpha expression. Continuous treatment with DFP results in inhibition of DPPIV enzyme activity in wtCD26 Jurkat transfectants (Figure 5B), associated with decreased expression of topoisomerase II alpha in these cells (Figure 5C). On the other hand, expression of topoisomerase II alpha in
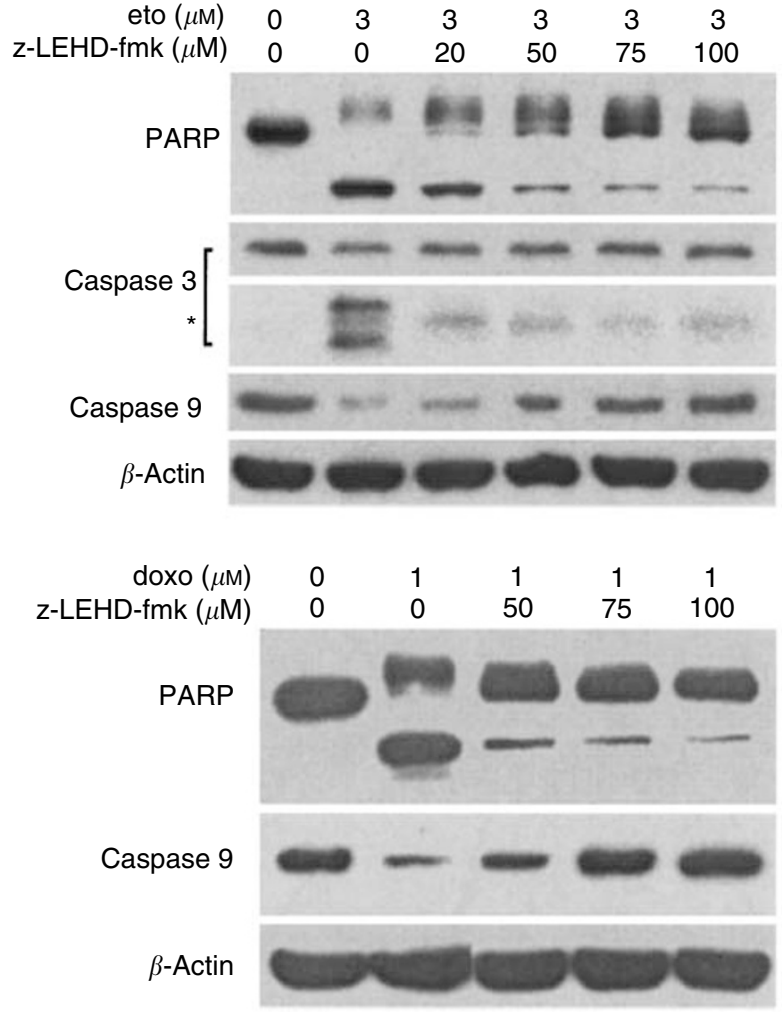

Figure 4 Effect of caspase-9 inhibitor z-LEHD-fmk on etoposideinduced apoptosis in wtCD26 Jurkat transfectant. wtCD26 Jurkat transfectants were incubated at $37^{\circ} \mathrm{C}$ for $2 \mathrm{~h}$ of preincubation with zLEHD-fmk at varying doses, and then treated with $3 \mu \mathrm{M}$ etoposide or I $\mu \mathrm{M}$ doxorubicin for $16 \mathrm{~h}$. Cells were then harvested, and whole-cell lysates were obtained as described in Materials and Methods. Following SDSPAGE of lysates, immunoblotting studies for PARP, caspase-3, caspase-9, and $\beta$-actin were performed as described in Materials and Methods (*): caspase- 3 cleaved products. Each lane was loaded with $30 \mu \mathrm{g}$ of protein.

parental Jurkat cells is not significantly affected by continuous exposure to DFP, as expected. Additionally, we examined the status of DPPIV enzyme activity and topoisomerase II alpha expression following DFP treatment. For this purpose, following treatment with DFP, wtCD26 cells were washed and incubated in culture media for the indicated time periods. We demonstrate that recovery of DPPIV enzyme activity is associated with recovery of topoisomerase II expression (Figure 5D and E). These results further corroborate and expand on our earlier findings regarding the importance of DPPIV enzyme activity in topoisomerase II alpha expression in CD26 Jurkat transfectants.

\section{Effect of soluble CD26 molecules on topoisomerase II alpha expression and sensitivity to doxorubicin}

It is theoretically possible that DPPIV effect is dependent on surface expression of the intact CD26/DPPIV molecule. To address this issue, we evaluate the effect of soluble CD26 (sCD26) molecules on topoisomerase II alpha expression. As shown in Figure 6, incubation with sCD26 molecules results in a significant increase in topoisomerase II alpha protein expression in parental control Jurkat cells (A) or Jiyoye cells (B). Along with an increase in topoisomerase II alpha expression, incubation of parental Jurkat cells with sCD26 molecules also results in enhanced doroxubicininduced or etoposide-induced PARP cleavage (Figure 7). Our findings further confirm that the presence of DPPIV activity itself, and not necessarily surface expression of CD26/DPPIV, augments 
A

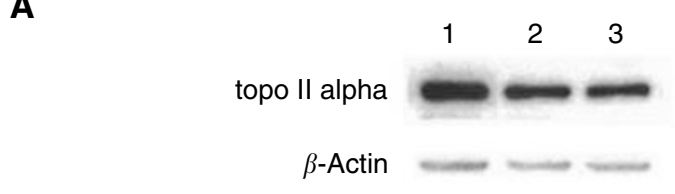

B
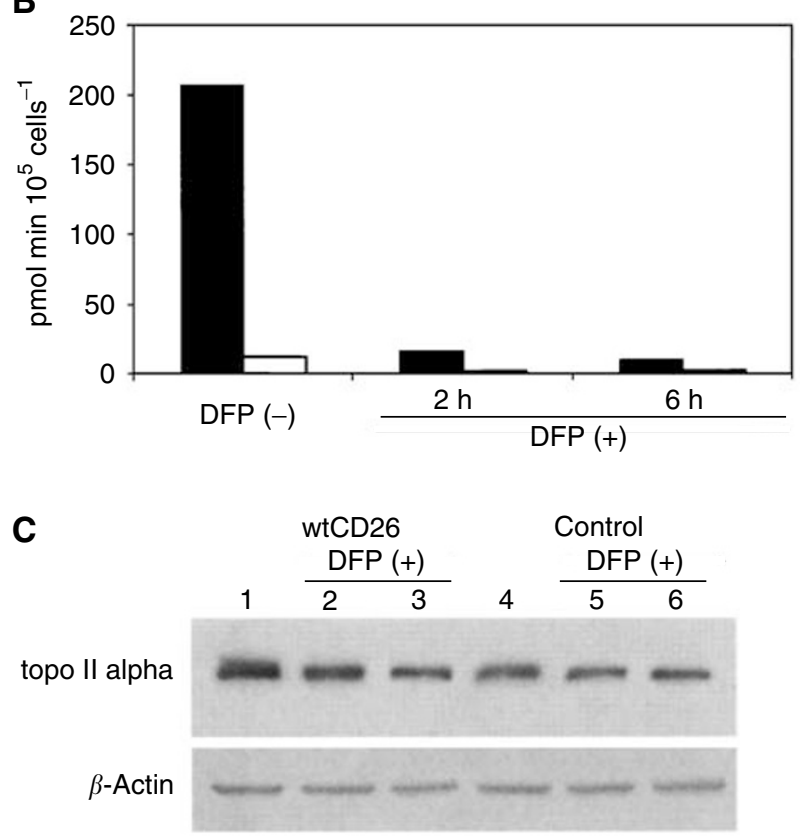

D

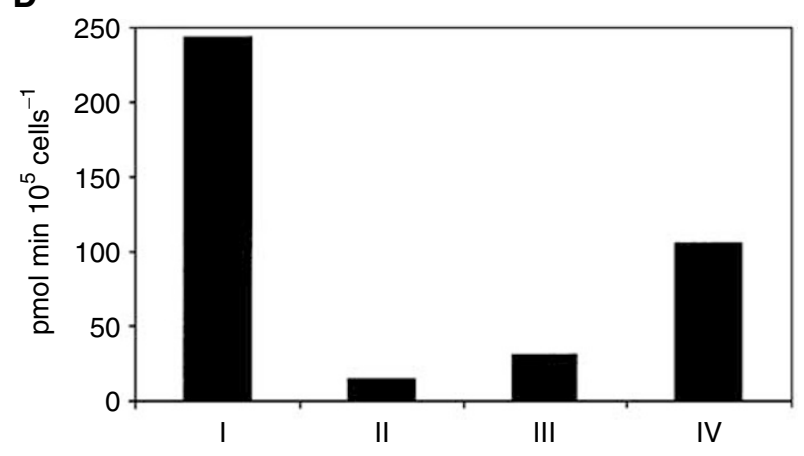

$\mathbf{E}$

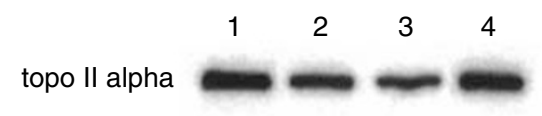

$\beta$-Actin

Figure 5 Effect of inhibition of DPPIV activity on topoisomerase II alpha expression. (A) After incubation of Jurkat cells at $37^{\circ} \mathrm{C}$ for $24 \mathrm{~h}$ in culture media, cells were harvested and nuclear extracts were obtained. Following SDS-PAGE of lysates, immunoblotting studies were performed for topoisomerase II alpha or $\beta$-actin as described in Materials and Methods. Each lane was loaded with $30 \mu \mathrm{g}$ of protein. Lane I: wtCD26 Jurkat transfectant, lane 2: S630A mutant transfectant, lane 3: parental Jurkat. (B) wtCD26 Jurkat transfectants or parental Jurkat were incubated in culture media alone (DFP-), culture media containing $100 \mu \mathrm{M}$ DFP for 2 or $6 \mathrm{~h}$ $(\mathrm{DFP}+)$. A representative sample of cells reflecting each treatment condition was obtained, and DPPIV enzyme activity assays were then performed as described in Materials and Methods. (C) wtCD26 Jurkat transfectants (lanes I-3) or parental Jurkat (lanes 4-6) were incubated in culture media alone (lanes I, 3), culture media containing $100 \mu \mathrm{M}$ DFP for $2 \mathrm{~h}$ (lanes 2, 5) or for $6 \mathrm{~h}$ (lanes 3, 6). Cells were harvested, and nuclear
A

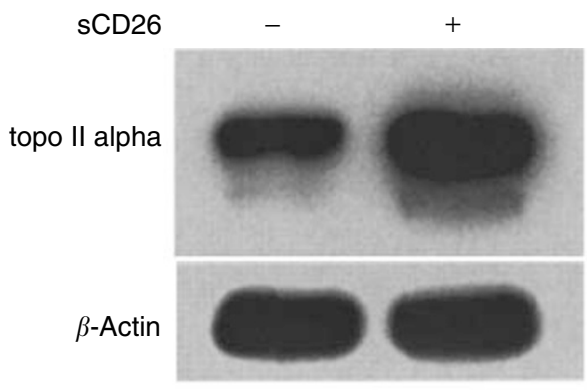

B

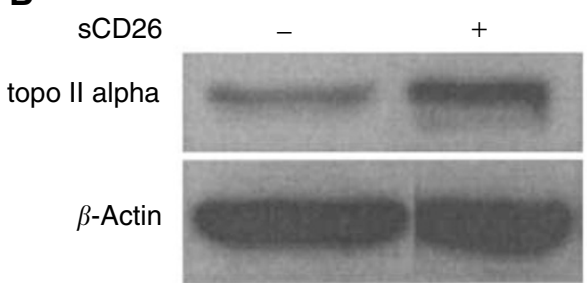

Figure 6 Effect of soluble CD26 molecules on topoisomerase II alpha expression. Parental Jurkat cells $(\mathbf{A})$ or liyoye cells $(\mathbf{B})$ were incubated overnight in culture media alone (-) or culture media containing soluble CD26 (sCD26) molecules $\left(300 \mu \mathrm{g} \mathrm{ml}{ }^{-1}\right)(+)$ at $37^{\circ} \mathrm{C}$. Cells were then harvested and nuclear extracts were obtained. Following SDS-PAGE of lysates, immunoblotting studies for topoisomerase II alpha or $\beta$-actin were performed as described in Materials and Methods. Each lane was loaded with $30 \mu \mathrm{g}$ of protein.

topoisomerase II alpha expression, leading to a resultant increase in sensitivity to topoisomerase II inhibitors.

\section{Caspase-9-dependent involvement of DR5 in etoposide- induced apoptosis in CD26 Jurkat transfectants}

Expression of death receptor 5 (DR5), a member of the TRAIL (tumour necrosis factor-related apoptosis-inducing ligand) family, is upregulated following treatment with such DNA-damaging agents as doxorubicin and etoposide (Gibson et al, 2000). We now demonstrate that etoposide treatment leads to a greater increase in the levels of the $58 \mathrm{kDa}$ DR5 in wtCD26 transfectants as compared with $5630 \mathrm{~A}$ or parental Jurkat cells (Figure $8 \mathrm{~A}$ ). Interestingly, Western blotting analyses with anti-DR $5 \mathrm{mAb}$ also detect the expression of a smaller $32 \mathrm{kDa}$ band with etoposide treatment, with

extracts were obtained. Following SDS-PAGE of lysates, immunoblotting studies for topoisomerase II alpha or $\beta$-actin were performed as described in Materials and Methods. Each lane was loaded with $30 \mu \mathrm{g}$ of protein. (D) wtCD26 Jurkat transfectants were incubated in culture media (bar I), or in culture media with $100 \mu \mathrm{M}$ DFP for $4 \mathrm{~h}$ (bar II), or they were incubated in culture media with $100 \mu \mathrm{M}$ DFP for $4 \mathrm{~h}$, then washed twice in PBS to ensure removal of DFP followed by incubation in culture media for $2 \mathrm{~h}$ (bar III) or $8 \mathrm{~h}$ (bar IV). A representative sample of cells reflecting each treatment condition was obtained, and DPPIV enzyme activity assays were then performed as described in Materials and Methods. (E) wtCD26 Jurkat transfectants were incubated in culture media (lane I), or in culture media with $100 \mu \mathrm{M}$ DFP for $4 \mathrm{~h}$ (lane 2), or they were incubated in culture media with $100 \mu \mathrm{M}$ DFP for $4 \mathrm{~h}$, then washed twice in PBS to ensure removal of DFP followed by incubation in culture media for $2 \mathrm{~h}$ (lane 3 ) or $8 \mathrm{~h}$ (lane 4 ). Cells were then harvested and nuclear extracts were obtained. Following SDS-PAGE of lysates, immunoblotting studies for topoisomerase II alpha or $\beta$-actin were performed as described in Materials and Methods. Each lane was loaded with $30 \mu \mathrm{g}$ of protein. 

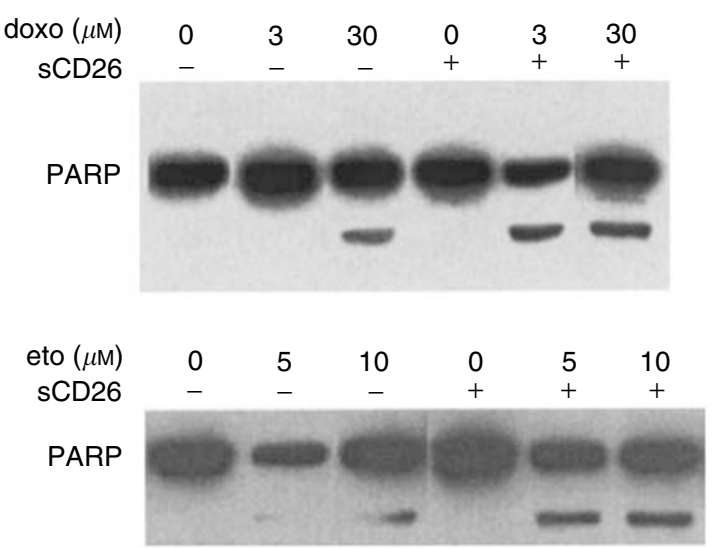

Figure 7 CD26-associated enhancement of doxorubicin or etoposideinduced PARP cleavage. Parental Jurkat cells were incubated overnight in culture media alone $(-)$ or culture media containing soluble CD26 $(\mathrm{sCD} 26)$ molecules $\left(300 \mu \mathrm{g} \mathrm{m}{ }^{-1}\right)(+)$ at $37^{\circ} \mathrm{C}$, followed by incubation with doxorubicin or etoposide at the indicated concentrations for $16 \mathrm{~h}$. Cells were then harvested, and whole-cell lysates were obtained as described in Materials and Methods. Following SDS-PAGE of lysates, immunoblotting studies for PARP or $\beta$-actin were performed as described in Materials and Methods. Each lane was loaded with $30 \mu \mathrm{g}$ of protein.

its levels again being significantly higher in wtCD26 Jurkat than S630A or parental cells. In time course studies, the appearance of the $32 \mathrm{kDa}$ band consistently precedes the observed increase in expression levels of the $58 \mathrm{kDa}$ band.

We have already demonstrated that etoposide-induced apoptosis in wtCD26 Jurkat transfectant involves caspase- 9 processing. To determine whether the enhancement in DR5 expression in etoposide-treated wtCD26 Jurkat is dependent on caspase-9related events, we examined DR5 expression in cells treated with etoposide following preincubation with the caspase-9-specific inhibitor z-LEHD-fmk. As demonstrated in Figure 8B, the increase in the $58 \mathrm{kDa}$ band seen in etoposide-treated wtCD26 cells is significantly attenuated when cells are preincubated with z-LEHDfmk. In addition, the $32 \mathrm{kDa}$ band induced by etoposide is no longer detectable with z-LEHD-fmk preincubation. Concordant with the findings with etoposide, pretreatment with z-LEHD-fmk similarly inhibits doxorubicin effect on DR5 status.

\section{DISCUSSION}

Through experiments involving CD26 Jurkat transfectants, DPPIV chemical inhibitor and soluble CD26/DPPIV molecules, we provide conclusive evidence that presence of DPPIV enzyme activity results in enhanced topoisomerase II alpha expression, associated with increased sensitivity to apoptosis induced by topoisomerase II inhibitors. Topoisomerase II enzyme plays an important role in the metabolism of DNA topoisomers and is essential for cellular proliferation (Wang, 1996). Two topoisomerase II isoforms, alpha and beta, exist in eukaryotes, coded by two different genes (Drake et al, 1989; Goswami et al, 1996). In particular, the $170 \mathrm{kDa}$ topoisomerase II alpha isoform is closely associated with the cell cycle, being highly expressed during cellular proliferation, and is the primary target of such topoisomerase II inhibitors as doxorubicin or etoposide (Burden and Osheroff, 1998). These drugs selectively exploit the catalytic activity of topoisomerase II alpha to create DNA damage by increasing the frequency and duration of DNA cleavage sites, causing permanent doublestranded breaks and leading to apoptosis (Froelich-Ammon and Osheroff, 1995; Beck et al, 1999; Mow et al, 2001). Owing to this mechanism of toxicity, increased enzyme level is associated with enhanced sensitivity, and drug resistance is related with reduced topoisomerase II alpha level (Beck et al, 1993, Oloumi et al, 2000). Our current findings that the enhanced topoisomerase II alpha expression associated with CD26/DPPIV presence results in greater

A

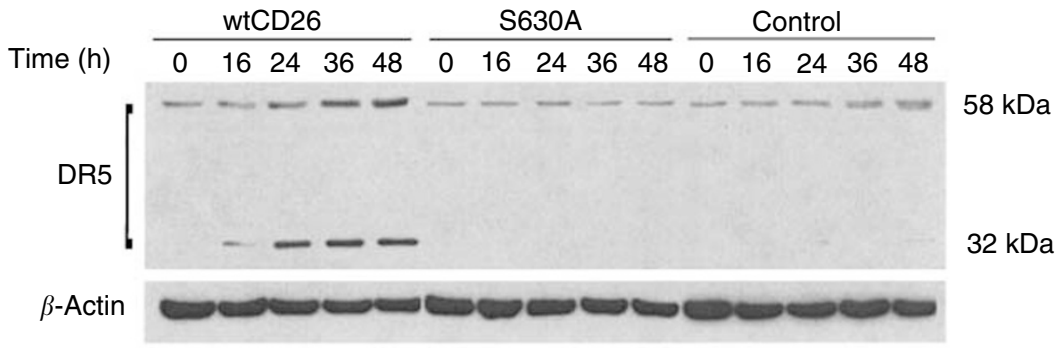

B

eto $(\mu \mathrm{M}) \quad 0 \quad 3 \quad 3 \quad 3$

Z-LEHD-fmk $(\mu \mathrm{M}) \quad 0 \quad 0 \quad 50100$

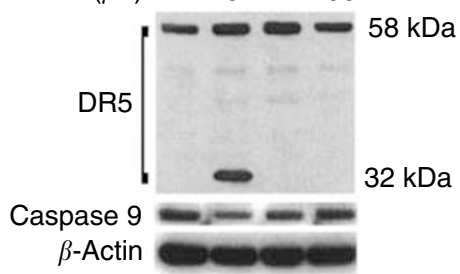

doxo $(\mu \mathrm{M})$ z-LEHD-fmk $(\mu \mathrm{m})$

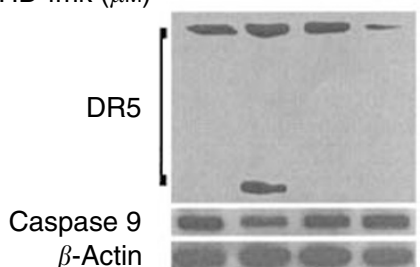

$58 \mathrm{kDa}$

$32 \mathrm{kDa}$

Figure 8 Effect of CD26/DPPIV on DR5 expression induced by etoposide treatment. (A) Jurkat cells were incubated at $37^{\circ} \mathrm{C}$ in culture media containing etoposide $(3 \mu \mathrm{M})$ for the indicated time periods at the indicated doses. Cells were then harvested, and whole-cell lysates were obtained as described in Materials and Methods. Following SDS-PAGE of lysates, immunoblotting studies for DR5 and $\beta$-actin were performed as described in Materials and Methods. Each lane was loaded with $30 \mu \mathrm{g}$ of protein. Anti- DR5 mAb detects two bands of 58 and $32 \mathrm{kDa}$. (B) Following $2 \mathrm{~h}$ of preincubation at $37^{\circ} \mathrm{C}$ with varying doses of z-LEHD-fmk, wtCD26 Jurkat transfectants were treated with $3 \mu \mathrm{M}$ etoposide or I $\mu \mathrm{M}$ doxorubicin for $48 \mathrm{~h}$. Cells were then harvested, and whole cell lysates were obtained as described in Materials and Methods. Following SDS-PAGE of lysates, immunoblotting studies for DR5, caspase-9, and $\beta$ actin were performed as described in Materials and Methods. Each lane was loaded with $30 \mu \mathrm{g}$ of protein. 
sensitivity to apoptosis induced by doxorubicin and etoposide are therefore consistent with the known mechanism of action of these topoisomerase II inhibitors.

Etoposide or doxorubicin engages the caspase-9-related mitochondrial pathway of apoptosis (Sun et al, 1999). It is also known that perturbation of Bcl-2-related proteins such as Bcl-xl is important for apoptotic processes associated with drug-induced DNA damage, potentially augmenting death signals from the mitochondrial pathway (Liu et al, 1996; Kluck et al, 1997; Fujita et al, 1998). Our results, including those demonstrating increased drug-induced Bcl-xl cleavage associated with CD26/DPPIV expression and the effect of the caspase-9-specific inhibitor z-LEHD-fmk, are consistent with the conclusion that the CD26/DPPIV-associated increase in apoptosis induced by the topoisomerase II inhibitors is mediated through caspase- 9 processing and the mitochondrial pathway. Nevertheless, it is theoretically possible that CD26 exerts its influence on cell growth inhibition via other additional pathway(s).

Our results also indicate that CD26/DPPIV expression is associated with enhancement of not only the $58 \mathrm{kDa}$ DR5 protein, but also the smaller $32 \mathrm{kDa}$ form following topoisomerase II inhibitor treatment of Jurkat cells. While being consistent with previous reports demonstrating that DR5 expression is upregulated following treatment with DNA-damaging agents (Gibson et al, 2000), our work also demonstrates the existence of the smaller $32 \mathrm{kDa}$ band. While the exact relationship between the $32 \mathrm{kDa}$ band and the full-length $58 \mathrm{kDa}$ band remains to be elucidated, one potential explanation from our work would be that the smaller band represents a precursor form of DR5. Our time course experiments show that while the $32 \mathrm{kDa}$ band is not detected in untreated cells, its appearance in cells treated with etoposide precedes the detectable increase in the expression levels of the $58 \mathrm{kDa}$ band. Additionally, the pretreatment with the caspase- 9 inhibitor $z$-LEHD-fmk completely inhibits topoisomerase II inhibitor-induced expression of the $32 \mathrm{kDa}$ band while abrogating the increased expression of the $58 \mathrm{kDa}$ band. Besides our demonstration of the existence of the $32 \mathrm{kDa}$ band, our work also reveals a functional relationship between caspase-9 and DR5. Previous work has indicated that death signals related with DR5 are subsequently transmitted downstream to caspase-9 processing events (Gibson et al, 2000). However, our findings suggest that caspase-9 processing also affects DR5 expression following drug-induced DNA damage, since pretreatment with the caspase- 9 inhibitor $z$-LEHD-fmk negatively affects expression levels of both the 58 and $32 \mathrm{kDa}$ bands in topoisomerase II inhibitor-treated cells.

Previously published work suggested that CD26/DPPIV expression renders human $\mathrm{T}$ cells more responsive to activation signals from various stimuli (Dang and Morimoto, 2002). In addition, CD26 expression on selected human tumours are associated with aggressive tumour behaviour (Carbone et al, 1995; Sato and Dang, 2003). Our present findings of the association between CD26/ DPPIV and topoisomerase II alpha expression may potentially provide an explanation for these previous observations. In view of the role played by topoisomerase II alpha in cellular proliferation, it is possible that the biological behaviour of these CD26-bearing cells reflects in part the higher levels of topoisomerase II alpha. Our present work thus provides additional evidence of the essential role of the multifaceted CD26 molecule in cellular processes. Furthermore, along with our recent study indicating an antitumour effect of anti-CD26 mAb (Ho et al, 2001), our findings may provide insights into the design of future novel treatments against selected human tumours based on our knowledge of CD26 biology.

\section{ACKNOWLEDGEMENTS}

NH Dang is supported by grants from the MD Anderson Cancer Center Physician-Scientist Program, the V Foundation, and the Gillson Longenbaugh Foundation. C Morimoto is supported by National Institutes of Health Grant AR33713. K. Sato is supported by a grant from the Eli Lilly Japan International Fellowship.

\section{REFERENCES}

Aytac U, Claret F-X, Ho L, Sato K, Ohmura K, Mills GB, Cabanillas F, Morimoto C, Dang NH (2001) Expression of CD26 and its associated depeptidyl peptidase IV enzyme activity enhances sensitivity to doxorubicin-induced cell cycle arrest at the G2/M checkpoint. Cancer Res 61: $7204-7210$

Aytac U, Sato K, Yamochi T, Ohnuma K, Mills GB, Morimoto C, Dang NH (2003) Effect of CD26/dipeptidyl peptidase IV on Jurkat sensitivity to G2/ $\mathrm{M}$ arrest induced by topoisomerase II inhibitors. Br J Cancer 88: $455-462$

Bauvois B, De Meester I, Dumont J, Rouillard D, Zhao HX, Bosmans E (1999) Costitutive expression of CD26/dipeptidyl peptidase IV on peripheral blood B lymphocytes of patients with B chronic lymphocytic leukeamia. Br J Cancer 79: $1042-1048$

Beck WT, Danks MK, Wolverton, JS, Kim R, Chen M (1993) Drug resistance associated with altered DNA topoisomerase II. Adv Enzyme Regul 33: $113-127$

Beck WT, Morgan SE, Mo YY, Bhat UG (1999) Drug resist. Tumor cell resistance to DNA topoisomerase II inhibitors: new developments. Drug Resist Update 2: $382-389$

Burden DA, Osheroff N (1998) Mechanism of action of eukaryotic topoisomerase II and drugs targeted to the enzyme. Biochim Biophys Acta 1400: 139 - 154

Carbone A, Gloghini A, Zagonel V, Aldinucci D, Gattei V, Degan M, Improta S, Sorio R, Monfardini S, Pinto A (1995) The expression of CD26 and CD40 ligand is mutually exclusive in human T-cell non-Hodgkin's lymphomas/leukemias. Blood 86: 4617-4626

Dang NH, Hafler DA, Schlossman SF, Breitmeyer JB (1990a) FcR-mediated crosslinking of Ta1 (CDw26) induces human T lymphocyte activation. Cell Immunol 125: $42-57$

Dang NH, Morimoto C (2002) CD26: an expanding role in immune regulation and cancer. Histol Histopathol 17: 1213-1226
Dang NH, Torimoto Y, Deusch K, Schlossman SF, Morimoto C (1990b) Comitogenic effect of solid-phase immobilized anti-1F7 on human CD4 T cell activation via CD3 and CD2 pathways. J Immunol 144: $4092-4100$

Dang NH, Torimoto Y, Schlossman SF, Morimoto C (1990c) Human CD4 helper $\mathrm{T}$ cell activation: functional involvement of two distinct collagen receptors, 1F7 and VLA integrin family. $J$ Exp Med 172: $649-652$

Dang NH, Torimoto Y, Shimamura K, Tanaka T, Daley JF, Schlossman SF, Morimoto C (1991) 1F7 (CD26): a marker of thymic maturation involved in the differential regulation of the CD3 and CD2 pathways of human thymocyte activation. J Immunol 147: 2825-2832

Dang NH, Torimoto Y, Sugita K, Daley JF, Schow P, Prado C, Schlossman SF, Morimoto C (1990d) Cell surface modulation of CD26 by anti-1F7 monoclonal antibody. Analysis of surface expression and human $\mathrm{T}$ cell activation. J Immunol. 145: 3963 - 3971

Drake FH, Hofmann GA, Bartus HF, Mattern MR, Crooke ST, Mirabelli CK (1989) Biochemical and pharmacological properties of p170 and p180 forms of topoisomerase II. Biochemistry 28: $8154-8160$

Froelich-Ammon SJ, Osheroff N (1995) Topoisomerase poisons: harnessing the dark side of enzyme mechanism. J Biol Chem 270: 21429-21432

Fujita N, Nagahashi A, Nagashima K, Rokudai S, Tsuruo T (1998) Acceleration of apoptotic cell death after the cleavage of Bcl-XL protein by caspase-3-like proteases. Oncogene 17: 1295-1304

Gibson SB, Oyer R, Spalding AC, Anderson SM, Johnson GL (2000) Increased expression of death receptor 4 and 5 synergizes the apoptosis response to combined treatment with etoposide and TRAIL. Mol Cell Biol 20: $205-212$

Goswami PC, Roti JL, Hunt CR (1996) The cell cycle-coupled expression of topoisomerase II alpha during $S$ phase is regulated by mRNA stability 
and is disrupted by heat shock or ionizing radiation. Mol Cell Biol 16: $1500-1508$

Haridas V, Higuchi M, Jayatilak GS, Bailey D, Mujoo K, Blake ME, Arntzen CJ, Gutterman JU (2001) Avicins: triterpenoid saponins from Acacia victoriae (Bentham) induce apoptosis by mitochondrial perturbation. Proc Natl Acad Sci USA 98: 5821 - 5826

Ho L, Aytac U, Stephens LC, Ohnuma K, Mills GB, McKee KS, Neumann C, LaPushin R, Cabanillas F, Abbruzzese JL, Morimoto C, Dang NH (2001) In vitro and in vivo antitumor effect of the anti-CD26 monoclonal antibody 1F7 on human CD30+ anaplastic large cell T-cell lymphoma Karpas 299. Clin Cancer Res 7: 2031-2040

Ishii T, Ohnuma K, Murakami A, Takasawa N, Kobayashi S, Dang NH, Schlossman SF, Morimoto C (2001) CD26-mediated signaling for T cell activation occurs in lipid rafts through its association with CD45RO. Proc Natl Acad Sci USA 98: 12138-12143

Jones D, Dang NH, Duvic M, Washington LT, Huh, YO (2001) Absence of CD26 expression is a useful marker for diagnosis of T-cell lymphoma in peripheral blood. Am J Clin Pathol 115: 885-892

Kajiyama H, Kikkawa F, Suzuki T, Shibata K, Ino K, Mizutani S (2002) Prolonged survival and decreased invasive activity attributable to dipeptidyl peptidase IV overexpression in ovarian carcinoma. Cancer Res 62: 2753-2757

Kluck RM, Bossy-Wetzel E, Green DR, Newmeyer DD (1997) The release of cytochrome $c$ from mitochondria: a primary site for Bcl-2 regulation of apoptosis. Science 275: $1132-1136$

Koreeda Y, Hayakawa M, Ikemi T, Abiko Y, Arch (2001) Isolation and characterisation of dipeptidyl peptidase IV from Prevotella loeschei ATCC 15930. Oral Biol 46: 759-766

Lauber K, Appel HA, Schlosser SF, Gregor M, Schulze-Osthoff K, Wesselborg S (2001) The adapter protein apoptotic protease-activating factor-1 (Apaf-1) is proteolytically processed during apoptosis. J Biol Chem 276: 29772-29781

Liu X, Kim CN, Yang J, Jemmerson R, Wang X (1996) Induction of apoptotic program in cell-free extracts: requirement for dATP and cytochrome $c$. Cell 86: $147-157$

Morimoto C, Torimoto Y, Levinson G, Rudd CE, Schrieber M, Dang NH, Letvin N, Schlossman SF (1989) 1F7, a novel cell surface molecule, involved in helper function of CD4 cells. J Immunol 143: 3430-3439

Morrison ME, Vijayasaradhi S, Engelstein D, Albino AP, Houghton AN (1993) A marker for neoplastic progression of human melanocytes in a cell surface ectopeptidase. J Exp Med 177: 1135-1143
Mow BM, Blajeski AL, Chandra J, Kaufmann SH (2001) Apoptosis and the response to anticancer therapy. Curr Opin Oncol 13: 453-462

Nishimura G, Proske RJ, Doyama H, Higuchi M (2001) Regulation of apoptosis by respiration: cytochrome $c$ release by respiratory substrates. FEBS Lett 505: 399-404

Oloumi A, MacPhail SH, Johnston PJ, Banath JP, Olive PL (2000) Changes in subcellular distribution of topoisomerase II alpha correlate with etoposide resistance in multicell spheroids and xenograft tumors. Cancer Res 60: $5747-5753$

Oravecz T, Pall M, Roderiquez G, Gorrell MD, Ditto M, Nguyen NY, Boykins R, Unsworth E, Norcross MA (1997) Regulation of the receptor specificity and function of the chemokine RANTES (regulated on activation, normal $\mathrm{T}$ cell expressed and secreted) by dipeptidyl peptidase IV (CD26)-mediated cleavage. J Exp Med 186: $1865-1872$

Perkins CL, Fang G, Kim CN, Bhalla KN (2000) The role of Apaf-1, caspase9 , and bid proteins in etoposide- or paclitaxel-induced mitochondrial events during apoptosis. Cancer Res 60: 1645-1653

Raynal P, Pollard HB (1994) Annexins: the problem of assessing the biological role for a gene family of multifunctional calcium- and phospholipid-binding proteins. Biochim Biophys Acta 1197: 63-93

Sato K, Dang NH (2003) CD26: a novel treatment target for T-cell lymphoid malignancies? Int J Oncol 22: 481 - 497

Sedo A, Revoltella RP (1995) Detection of dipeptidyl peptidase IV in glioma $\mathrm{C} 6$ and neuroblastoma SK-N-SH cell lines. Biochem Cell Biol 73. $113-115$

Stecca BA, Nardo B, Chieco P, Mazziotti A, Bolondi L, Cabaralli AJ (1997) Aberrant dipeptidyl peptidase IV (DPPIV/CD26) expression in human hepatocellular carcinoma. Hepatology 27: 337-345

Sun XM, MacFarlane M, Zhuang J, Wolf BB, Green DR, Cohen GM (1999) Distinct caspase cascades are initiated in receptor-mediated and chemical-induced apoptosis. J Biol Chem 274: 5053-5060

Tanaka T, Duke-Cohan JS, Kameoka J, Yaron A, Lee I, Schlossman SF, Morimoto C (1994) Enhancement of antigen-induced T-cell proliferation by soluble CD26/dipeptidyl peptidase IV. Proc Natl Acad Sci USA 91: $3082-3086$

Tanaka T, Umeki K, Yamamoto I, Sakamoto M, Noguchi S, Ohtaki S (1995) CD26 (dipeptidyl peptidase IV/DPPIV) as a novel molecular marker for differentiated thyroid carcinoma. Int $I$ Cancer 64: $326-333$

Wang JC (1996) DNA topoisomers. Annu Rev Biochem 65: 635-692 